\title{
A TAT-DEF-Elk-1 Peptide Regulates the Cytonuclear Trafficking of Elk-1 and Controls Cytoskeleton Dynamics
}

\author{
Jérémie Lavaur, ${ }^{1,3}$ Frédéric Bernard, ${ }^{2,3}$ Pierre Trifilieff, ${ }^{1,3,4,5}$ Vincent Pascoli, ${ }^{1,3}$ Vincent Kappes, ${ }^{1,3}$ Christiane Pagès, ${ }^{1,3}$ \\ Peter Vanhoutte, ${ }^{1,3}$ and Jocelyne Caboche ${ }^{1,3}$ \\ ${ }^{1}$ Signalisation Neuronale et Régulations Géniques and ${ }^{2}$ Développement Neuronal, Centre National de la Recherche Scientifique (CNRS), Unité Mixte de \\ Recherche (UMR) 7102, 75005 Paris, France, ${ }^{3}$ Université Pierre et Marie Curie-Paris 6, 75005 Paris, France, ${ }^{4}$ CNRS, UMR 5106, 33405 Talence, France, and \\ ${ }^{5}$ Université Bordeaux I, 33405 Talence, France
}

The transcription factor Elk-1 plays a key role in cell differentiation, proliferation and apoptosis. This role is thought to arise from its phosphorylation by activated extracellular signal-regulated kinases (ERKs), a critical posttranslational event for the transcriptional activity of the ternary complex composed of Elk-1 and a dimer of serum response factor (SRF) at the serum response element (SRE) regulatory site of transcription. In addition to its nuclear localization, Elk-1 is found in the dendrites and soma of neuronal cells and recent evidence implicate a cytoplasmic proapoptotic function of Elk-1, via its association with the mitochondrial permeability transition pore complex. Thus, the nuclear versus cytoplasmic localization of Elk-1 seems to be crucial for its biological function. In this study we show that the excitatory neurotransmitter, glutamate, induces an ERK-dependent Elk-1 activation and nuclear relocalization. We demonstrate that Elk-1 phosphorylation on Ser383/389 has a dual function and triggers both Elk-1 nuclear translocation and SRE-dependent gene expression. Mutating these sites into inactive residues or using a synthetic penetrating peptide (TAT-DEF-Elk-1), which specifically interferes with the DEF docking domain of Elk-1, prevents Elk-1 nuclear translocation without interfering with ERK nor MSK1 (mitogenand stress-activated protein kinase 1), a CREB kinase downstream from ERK- activation. This results in a differential regulation of glutamate-induced IEG regulation when compared with classical inhibitors of the ERK pathway. Using the TAT-DEF-Elk-1 peptide or the dominant-negative version of Elk-1, we show that Elk-1 phosphorylation controls dendritic elongation, SRF and Actin expression levels as well as cytoskeleton dynamics.

Key words: extracellular signal-regulated kinase (ERK); Elk-1; Elk-1 inhibitor; trafficking; gene regulations; neuronal differentiation; TAT-DEF-Elk-1 peptide

\section{Introduction}

The transcription factor Elk-1 represents a major nuclear target of mitogen-activated protein kinases (MAPK). It belongs to the ternary complex factor (TCFs) family of ETS-domain transcription factors, which also includes SAP-1 and NET/ERP/SAP-2/ Elk-3 (Buchwalter et al., 2004). TCFs phosphorylation drives the rapid transcription of immediate early genes (IEG) through a ternary complex formed with a dimer of serum response factor (SRF) on the serum response element (SRE) DNA regulatory site. In addition to their N-terminal ETS DNA-binding domain, TCFs contain a "B box" mediating their interaction with SRF, a $\mathrm{C}$-terminal transactivation domain encompassing the residues

\footnotetext{
Received May 18, 2007; revised 0ct. 29, 2007; accepted Nov. 6, 2007.

This work was supported by the Centre National de la Recherche Scientifique, the Agence Nationale pour la Recherche, the Fondation pour la Recherche Médicale (FRM), and Fondation Jérôme Lejeune. J.L. was supported by the Edmond Rothschild Chemical Dependency Institute Beth Israell Medical Center, V.P. by FRM, and P.T. by the Fondation Jérome Lejeune. We thank Dr. Virginie Georget and Richard Schwartzmann (Institut Federatif de Recherche de Biologie Integrative, IFR-83) for their assistance with confocal microscopy. The TAT-DEF-Elk-1 peptide has been patented: EP 05290363 and W0 2006/087242.

Correspondence should be addressed to Dr. Jocelyne Caboche, Université Pierre et Marie Curie-Paris 6, Centre National de la Recherche Scientifique, Unité Mixte de Recherche 7102, 9 quai Saint Bernard, 75005 Paris, France. E-mail: jocelyne.caboche@snv.jussieu.fr.

DOl:10.1523/JNEUROSCI.2279-07.2007

Copyright $\odot 2007$ Society for Neuroscience $\quad$ 0270-6474/07/2714448-11\$15.00/0
}

phosphorylated by MAPK and two docking domains, the D (or DEJL, for docking site for ERK and JNK, LXL) and the FxFP (or DEF for docking site for ERK, FXF) domains and act as docking sites for MAPK that are crucial for the recognition and interaction with activated MAPK (Sharrocks et al., 2000).

In cell lines, Elk-1 has a nuclear expression which relies on a nuclear localization signal (NLS) present within its ETS domain (Janknecht et al., 1994). Once phosphorylated on Ser383/Ser389, Elk-1 undergoes conformational changes leading to increased DNA-binding and transcriptional activity (Yang et al., 1999). In neuronal cells, we reported that Elk-1 was expressed in both nuclear and cytoplasmic compartments (Sgambato et al., 1998a; Vanhoutte et al., 2001). This suggested that Elk-1 may have additional functions outside the nucleus. More recently, it was shown that Elk-1 plays important extranuclear functions, because it can be associated with the mitochondrial permeability transition pore complex (PTP) where it plays a proapoptotic, role (Barrett et al., 2006a). The posttranslational SUMOylation of Elk-1 controls its nucleus-to-cytoplasm shuttling. Mutating the critical residues for Elk-1 SUMOylation resulted in a faster shuttling of Elk-1 to the nucleus, an effect associated with neuronal differentiation in PC12 cells (Salinas et al., 2004). Thus, the cytoplasm-to-nucleus shuttling of Elk-1 seems to be important 
for deciphering its role in cell survival and/or neuronal differentiation.

During electrical stimulation of the corticostriatal pathway (Sgambato et al., 1998a), glutamate application in striatal slices (Vanhoutte et al., 1999) or cocaine administration (Valjent et al., 2000), Elk-1 becomes phosphorylated by the MAPK/ERK in dendritic, cytoplasmic and nuclear compartments, raising the possibility that Elk-1 activation occurs primarily in dendrites and triggers its translocation to the nucleus in which its controls transcription and long-term neuronal adaptations. Herein, we show that ERK-dependent Elk-1 phosphorylation is necessary and sufficient to drive Elk-1 nuclear translocation and subsequent SRE-driven gene expression. A cell penetrating peptide (TAT-DEF-Elk-1), which mimics the DEF domain of Elk-1, does not interfere with ERK activation but blocks Elk-1 phosphorylation and nuclear translocation as well as SRE-dependent gene regulation. As opposed to classical ERK inhibitors, TAT-DEFElk-1 preserves ERK activity toward other substrates. Finally, inhibiting Elk-1 phosphorylation using this inhibitor peptide or overexpression of a nonphosphorylatable version of Elk-1 impairs dendritic elongation in vitro. We conclude that Elk-1 phosphorylation has a dual role in neuronal cells by controlling its nuclear localization and role in transcription. These molecular events are critically involved in neuronal differentiation.

\section{Materials and Methods}

Animals and drug administration. Male C57BL/6J mice (10 weeks old) were purchased from Janvier (Le Genest St. Isle, France). The animals were housed in standard cages with ad libitum access to food and water with a 12/12-h light/dark cycle. All experiments took place during the animal's light cycle. Three days before the experiments, mice were handled for 2-3 min/d. Cocaine ( $20 \mathrm{mg} / \mathrm{kg}$; Sigma-Aldrich, St. Louis, MO) was injected intraperitoneally and dissolved in $0.9 \% \mathrm{NaCl}$. The mitogen activated and extracellular-regulated protein kinase (MEK) inhibitor $\alpha$-[amino[(4-aminophenyl)thio]methylene]-2(trifluoromethyl)-

benzene acetonitrile (SL327) was dissolved in DMSO, diluted twice in sterile water, and injected $(50 \mathrm{mg} / \mathrm{kg}) 1 \mathrm{~h}$ before cocaine administration. Animal care was conducted in accordance with the standard ethical guidelines (European Communities Guidelines on the Care and Use of Laboratory Animals: 86/609/EEC).

Neuronal cell cultures. Striata were dissected out from 14-d-old Swiss mouse embryos (Janvier, France) and prepared as previously described (Brami-Cherrier et al., 2002). Cells were suspended in Neurobasal medium supplemented with B27 (Invitrogen, Cergy Pontoise, France), 500 nM L-glutamine, $60 \mu \mathrm{g} / \mathrm{ml}$ penicillin $\mathrm{G}$ and $25 \mu \mathrm{M} \beta$-mercaptoethanol (Sigma, L'Isle d'Abeau, Chasnes, France) and plated into 24-well $(1.8 \times$ $10^{5}$ cells per well) or 6 -well $\left(8.6 \times 10^{5}\right.$ cells per well) Nunc (Dutscher, Brumath, France) multiwell plates coated with $50 \mu \mathrm{g} / \mathrm{ml}$ poly-D-lysine (Sigma). After $3 \mathrm{~d}$ in vitro (DIV 3), Ara C (cytosine arabinoside, $2.5 \mu \mathrm{M}$; Sigma-Aldrich) was added to prevent astrocytes proliferation. The day before the experiment, neurons were put back in Neurobasal medium without Ara C. For all experiments, treatments were performed between DIV 7 and DIV 9, when the majority of cells were of neuronal phenotype with no detectable glial elements. For pharmacological treatments, the various drugs used (see below, Chemical and reagents) were added directly to the medium, and the cells were replaced at $37^{\circ} \mathrm{C}$ in a humidified atmosphere of $95 \%$ air and $5 \% \mathrm{CO}_{2}$ for the appropriate time.

cDNA constructs and transfections. Three different cDNAs were used (Vanhoutte et al., 2001): a dominant-negative Elk-1 (HA-DN-Elk-1, corresponding to $\mathrm{Ser}^{383} / \mathrm{Ala}^{383}$ and $\mathrm{Ser}^{389} / \mathrm{Ala}^{389}$ mutations), a constitutively active version of Elk-1 (HA-CA-Elk-1, corresponding to Ser ${ }^{383}$ / $\mathrm{Asp}^{383}$ and $\mathrm{Ser}^{389} / \mathrm{Asp}^{389}$ mutations) and a wild-type version (HA-WtElk-1). These constructs were cloned in pcDNA3-HA and pAcmCHERRY-N2 expression vectors. Transient transfections of primary striatal cultures with GFP or HA-tagged Elk-1 plasmids were performed using Lipofectamine 2000 (Invitrogen) according to the manu- facturer's instructions. Cells $\left(600 / \mathrm{mm}^{2}\right)$ were transfected with $1 \mu \mathrm{g}$ of DNA for $3.5 \mathrm{~h}$. Neurons were then rinsed with fresh culture medium and put back to $37^{\circ} \mathrm{C}$ for $24 \mathrm{~h}$ before pharmacological treatments. For HAtagged versions, expression of Elk-1 constructs in transfected neurons was visualized by immunodetection of the HA tag using a monoclonal anti-HA antibody (1:250; Roche Diagnostics, Indianapolis, IN) or a polyclonal anti-HA antibody (1:250; Zymed Laboratories, Invitrogen) and revealed with an anti-mouse FITC-labeled secondary antibody [1:100; Jackson ImmunoResearch, Beckman Coulter (Marseille, France)] or anti-rabbit FITC-conjugated antibody (1:100; Sigma-Aldrich), respectively.

Chemical and reagents. Primary striatal neurons were incubated with $10 \mu \mathrm{M}$ U0126 (Calbiochem, San Diego, CA) for $30 \mathrm{~min}$ before and during treatment with L-glutamic acid (100 $\mu \mathrm{M}$; Calbiochem). The unconjugated and FITC-coupled forms of the TAT-DEF-Elk-1 (GRKKRRQRRRPPSPAKLSFQFPSSGSAQVHI) as well as the scramble (GRKKRRQRRRPPQSKPSGSQHPIFSLAFVAS) peptides were synthesized by Genosphere Biotechnologies (Paris, France) and applied to striatal neurons during $1 \mathrm{~h}$. To remove the excess of peptide, neurons were washed once with culture media without peptide and put back at $37^{\circ} \mathrm{C}$ for 30 min before glutamate treatment.

Immunocytochemistry. After the appropriate time of glutamate treatment, cells were fixed with PBS containing 4\% paraformaldehyde (PFA) (added directly to the medium to a final concentration of $2 \%$ ) for $40 \mathrm{~min}$ at room temperature (RT) and then incubated with methanol/acetone solution (50:50) for $10 \mathrm{~min}$ at $4^{\circ} \mathrm{C}$. After three rinses in PBS, cells were treated with blocking buffer (normal goat serum 10\% in PBS) for $2 \mathrm{~h}$ at RT (except for P-ERK and P-H3 staining in which the blocking buffer contained $10 \%$ fetal calf serum, $1 \%$ BSA in PBS). Antibodies raised against c-Fos (1:7000; Santa Cruz Biotechnology, Tebu, Le Perray-enYvelines, France), zif 268 (1:2000 in 5\% NGS; Santa Cruz Biotechnology), junB (1:500; Santa Cruz Biotechnology), c-jun (1:200; Cell Signaling Technology, Ozyme, Saint Quentin-en-Yvelines, France), MAP-2 (1:1000; Sigma-Aldrich), Elk-1 (1:500; Santa Cruz Biotechnology), phospho-Ser ${ }^{383}$ Elk-1 (1:250; Santa Cruz Biotechnology), phospho-Thr ${ }^{581}$ MSK-1 (mitogen- and stress-activated protein kinase 1; 1:750; Cell Signaling Technology), phospho-Ser ${ }^{133}$ CREB (1:2000; Upstate Biotechnology) were incubated overnight (O.N) at $4^{\circ} \mathrm{C}$ diluted in PBS or in PBS containing $1 \%$ BSA, $0.05 \%$ Tween 20 for P-ERK (dually phosphorylated ERK (Thr ${ }^{202}-\mathrm{Tyr}^{204} \mathrm{p} 44 / 42$ MAPK; 1:500; Cell Signaling Technology) and P-H3 (anti-phospho-Ser ${ }^{10}-\mathrm{H} 3$; 1:1000; Upstate Biotechnology) immunocytochemistry. Neurons were then incubated with secondary Cy3conjugated anti-rabbit (1:2000; GE Healthcare, Little Chalfont, UK) or anti-mouse antibodies (1:2000; Jackson ImmunoResearch). For some experiments, a FITC-coupled anti-rabbit antibody (1:100; Jackson ImmunoResearch) was used for $2 \mathrm{~h}$ at RT. After rinses, cells were counterstained with Hoechst (Invitrogen) and mounted under coverslips using Vectashield (Vector Laboratories, Abcys, Paris France). For double labeling experiments and confocal analysis, anti-mouse Alexa Fluor 488 (1: 400), anti-mouse Alexa Fluor 546 (1:400), and anti-rabbit Alexa Fluor 568 (1:200; Invitrogen) were used.

Western blots. After pharmacological treatments for the appropriate time, the culture medium was quickly removed and ice-cold lysis buffer containing a mix of protease and phosphatase inhibitors was added to striatal cells $(10 \mathrm{~mm}$ Tris- $\mathrm{HCl}, 30 \mathrm{~mm}$ sodium pyrophosphate, $50 \mathrm{~mm}$ $\mathrm{NaCl}, 1 \%$ Triton X-100, $50 \mathrm{~mm} \mathrm{NaF}, 5 \mu \mathrm{M} \mathrm{ZnCl}, 100 \mu \mathrm{M} \mathrm{Na} \mathrm{VO}_{4}, 1 \mathrm{~mm}$ DTT, $100 \mathrm{~nm}$ okadaic acid, $2.5 \mu \mathrm{g} / \mathrm{ml}$ aprotinin, $2.5 \mu \mathrm{g} / \mathrm{ml}$ pepstatin, 0.5 mM PMSF, $0.5 \mathrm{~mm}$ benzamidine, $2.5 \mu \mathrm{g} / \mathrm{ml}$ leupeptin). Dishes were rapidly placed on dry ice, scraped, lysed for $10 \mathrm{~min}$ on ice and vortexed for 1 min. Insoluble material was removed by centrifugation $(13,000 \times g ; 20$ $\left.\min ; 4^{\circ} \mathrm{C}\right)$. Thirty micrograms of proteins were analyzed by SDS-PAGE on $10 \%$ gels as described by Vanhoutte et al. (1999). After blocking of nonspecific sites with $5 \%$ nonfat dry milk, blots were incubated O.N at $4{ }^{\circ} \mathrm{C}$ with primary antibodies diluted in blocking solution with $0.1 \%$ Tween 20 [(1:1000, phospho-CREB; Upstate Biotechnology) (1:250, phospho-Elk-1; Santa Cruz Biotechnology) (1:2500, phosphorylated ERK Thr ${ }^{202}-\mathrm{Tyr}^{204}$ p44/42 MAPK; Cell Signaling Technology) (1:500, phospho-MSK-1; Cell Signaling Technology) (1:200, SRF; Santa Cruz Biotechnology) (1:10,000, actin; Sigma-Aldrich) (1:5000, $\beta$-tubulin; 
Sigma-Aldrich)]. Blots were then washed and incubated for $1 \mathrm{~h}$ at RT with anti-rabbit (1:5000; GE Healthcare) or anti-mouse horseradish peroxidase (HRP)-conjugated antibodies diluted (1:5000; GE Healthcare) in the blocking solution with $0.1 \%$ Tween 20 . Proteins were detected by chemiluminescence using the ECL kit (GE Healthcare).

Subcellular fractionation. Neurons were placed on ice and scraped gently in an ice-cold hypotonic lysis buffer [(HEPES 5 mM, pH 7.9, $\mathrm{MgCl}_{2} 1.5 \mathrm{~mm}, \mathrm{KCl} 10 \mathrm{~mm}$, Triton X-100 $0.125 \%$ and a mix of protease and phosphatase inhibitors (see above, Western blots)]. Lysates were incubated on ice during $10 \mathrm{~min}$ and centrifuged at $4000 \times g$ for $10 \mathrm{~min}$ at $4^{\circ} \mathrm{C}$. The soluble fraction, corresponding to the cytoplasm, was collected and kept on ice. The pellet, corresponding to the nuclear fraction, was washed twice with the hypotonic buffer and incubated for $30 \mathrm{~min}$ at $4^{\circ} \mathrm{C}$ in a hypertonic buffer (HEPES $20 \mathrm{~mm}$, glycerol 25\%, $\mathrm{NaCl} 0.5 \mathrm{~mm}$, $\mathrm{MgCl}_{2} 0.5 \mathrm{~mm}$, EDTA $0.5 \mathrm{~mm}$, Triton X-100 $0.25 \%$ ) and a mix of protease and phosphatase inhibitors. The resulting cytoplasmic and nuclear fractions were then sonicated and subjected to SDS-PAGE electrophoresis.

Neuronal differentiation. When indicated, primary striatal neurons were incubated with $10 \mu \mathrm{M}$ U0126, $10 \mu \mathrm{M}$ DMSO (Calbiochem), or 5 $\mu \mathrm{M}$ TAT-DEF-Elk-1 (D enantiomer form) at DIV 3 and left in the medium containing the various drugs until DIV 7. At DIV 6, striatal neurons were transfected with a cDNA encoding for GFP to visualize the whole neuronal morphology. At DIV7, neurons were fixed with paraformaldehyde, and a Map-2 staining was performed to visualize dendrites. Constructs encoding mCherry-DN-Elk-1 or -CA-Elk-1 were cotransfected with GFP at DIV 1. Randomly selected photographs were taken (magnification, 20X) with a minimum of 20 pictures per condition. For each transfected neuron, both axonal and dendritic length was determined on the basis of GFP staining using a computer-assisted image analyzer (UTHSCSA Image Tool 3.0). Dendrites were determined based on MAP2 staining and axons as the longest neuritic extension. The mean dendritic length was measured after calculation of the number of processes (magnification, $40 \times)$. A minimum of 20 fields ( $~ 50-60$ transfected neurons) was analyzed for each experimental condition. The actin cytoskeleton was visualized by a one-step fixation/permeabilization and staining process consisting of the addition of $4 \%$ formaldehyde with TRITC-labeled phalloidin (Invitrogen) for $20 \mathrm{~min}$ followed by a classical $\beta$-tubulin (1: 500; Sigma-Aldrich) immunofluorescent staining (FITC).

Quantifications and statistical analysis. Pictures of immunostaining were taken with a Leica DM LB (Rueil-Malmaison, France) fluorescence microscope coupled to a CCD. Images from immunofluorescence were taken (magnification, $20 \times$ ) in parallel with Hoechst staining, from 20 independent fields for each experiment $(n=3$ independent experiment for each treatment). The number of immunopositive cells was determined using Image Pro Plus 4.5.0.19 software (Media Cybernetics, Wokingham, Berkshire, UK) after setting an intensity threshold applied automatically to all the pictures taken within an experiment. The percentage of immunopositive cells was defined as the number of immunoreactive cells over the number of Hoechst-stained neurons.

For Western blot experiments, films were scanned and analyzed using Scion Image software. Relative kinase and transcription factor expression level or activation was measured by normalization of the density obtained from the protein of interest with a protein used as loading control ( $\beta$-tubulin) or GAPDH.

Data were expressed as mean \pm SEM, and significance was determined by unpaired Student's $t$ test. In all cases, significance was set at $p<0.05$.

\section{Results}

ERK activation drives nuclear translocation of Elk-1 in striatal neurons in vivo and in vitro

We first wished to analyze whether Elk-1 might translocate to the nucleus after activation. To address this, we used a well established model system of Elk-1 phosphorylation in vivo. During cocaine administration, we have shown a rapid activation of ERK along with Elk-1 within the striatum (Valjent et al., 2000) (Fig. $1 A)$. In this model, we analyzed the subcellular localization of Elk-1 by immunohistochemistry and found it was enriched in the cytoplasm, including neurites, in saline-treated striatal neurons (Fig. $1 B$ ). However, during cocaine stimulation, Elk-1 showed a strictly nuclear expression pattern (Fig. $1 B$ ). The nuclear expres- 
A

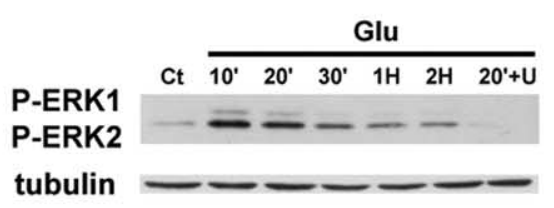

B

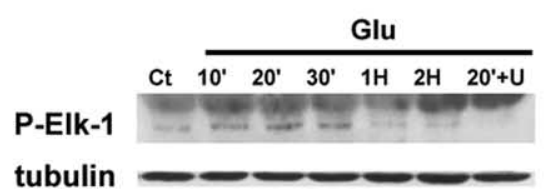

C

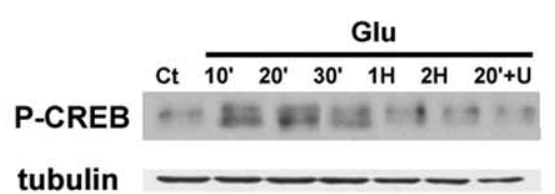

D

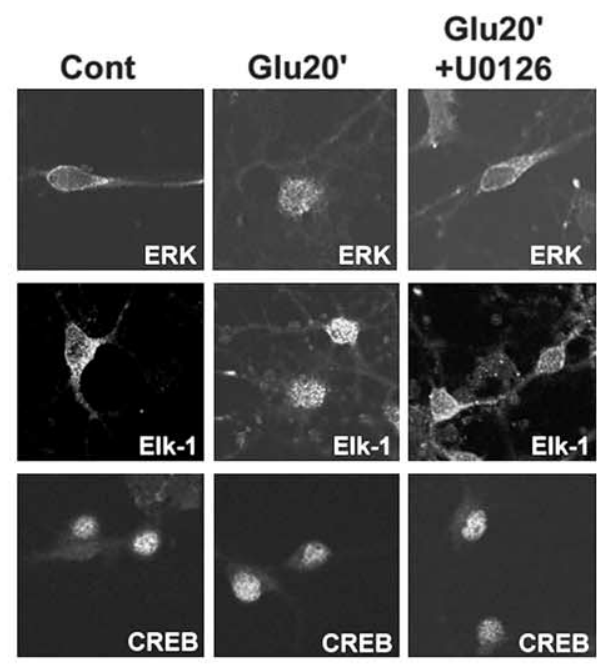

E

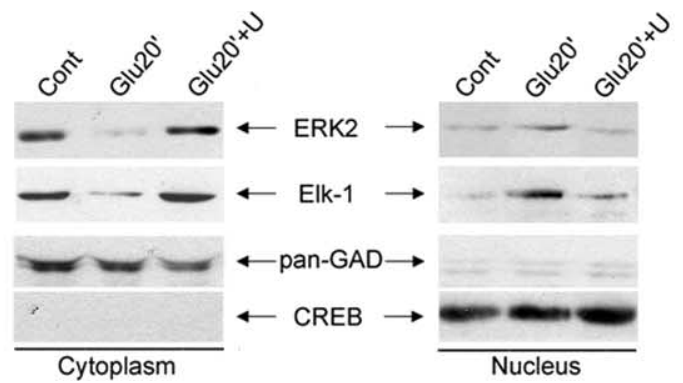

Figure 2. Nuclear translocation of Elk-1 depends on ERK activation by glutamate in vitro. Striatal neurons were incubated with $100 \mu \mathrm{m}$ glutamate (Glu) for 10, 20, and $30 \mathrm{~min}$ and 1 and $2 \mathrm{~h}$. When indicated, the selective MEK inhibitor U0126 (10 $\mu \mathrm{M})$ was applied 30 min before and during glutamate application $\left(20^{\prime}+U\right)$. Western blot analysis of ERK1/2 (A), Elk-1 (B), and CREB (C) activation were performed with antiphospho-(Thr $\left.{ }^{202}-\operatorname{Tyr}^{204}\right)$-ERK1/2, antiphospho-(Ser $\left.{ }^{383}\right)$-Elk-1, and antiphospho-(Ser $\left.{ }^{133}\right)$ CREB antibodies, respectively. As a loading control, the same blots were probed with a $\beta$-tubulin antibody. Right column illustrates the quantifications of ERK2 $(\boldsymbol{A})$, Elk-1 $(\boldsymbol{B})$, and CREB $(\boldsymbol{C})$ activation analyzed from three independent experiments by measuring the intensity of the phospho-protein signal normalized to the $\beta$-tubulin staining. Statistical analyses were performed between controls and glutamate-treated samples using unpaired Student's $t$ test, ${ }^{*} p<0.05 ;{ }^{* *} p<0.005 ;{ }^{* * *} p<0.001 ;{ }^{\# \#} p<$ 0.005 when comparing glutamate $20^{\prime}+U$ with glutamate $20^{\prime}$ alone. $\boldsymbol{D}$, Confocal analysis of endogenous ERK (top row), Elk- 1 (middle row), and CREB (bottom row) expression in control conditions (Cont), during 20 min of glutamate treatment (Glu20'), or sion of Elk-1 was abolished in mice treated with SL327, a selective MEK inhibitor that crosses the blood-brain barrier (Atkins et al., 1998), administered before cocaine. Colabeling of phosphorylated Elk-1 and Elk-1 in cocaine-treated striata, clearly showed an enrichment of nuclear Elk-1 in neurons in which Elk-1 was activated (Fig. 1C). Thus, altogether these data strongly support that Elk-1 translocates from the cytoplasm to the nucleus during phosphorylation by activated ERKs.

To determine the molecular mechanisms responsible for Elk-1 nuclear translocation, and analyze the role of its phosphorylation in this translocation, we set up an in vitro model whereby glutamate activates ERK along with Elk-1 in striatal neurons in culture (Fig. 2A,B). This model system also leads to the activation of the transcription factor CREB (Fig. 2C), as previously described during glutamate stimulation of striatal slices (Vanhoutte et al., 1999). Glutamate-induced Elk-1 and CREB phosphorylation were totally dependent on ERK activation, as shown in experiments in which the selective MEK inhibitor U0126 was applied before glutamate (Fig. $2 A-C$ ).

On this in vitro model system, we then analyzed cytoplasmic versus nuclear expression patterns of ERKs and Elk-1. This was performed by confocal analyses of total ERKs and Elk-1 expression (Fig. 2D), and Western blot detection of these proteins after nuclear/cytoplasmic fractionation (Fig. 2E). During glutamate treatment, we found an enrichment of total ERK proteins in the nuclear compartment, with also a preferential nuclear localization of total Elk-1 proteins. In contrast, CREB and GAD proteins, which were used as nuclear and cytoplasmic markers, respectively, did not show any shuttling between the cytoplasm and the nucleus, whatever the treatment (Fig. 2D, bottom, $E$ ). Pretreatment of striatal neurons with the MEK inhibitor, U0126, before gluta-

$\leftarrow$

glutamate in the presence of U0126 treatment (Glu20' + U0126). Note that ERK and Elk-1 proteins are present in somatic and dendritic compartments in control conditions and translocate to the nucleus in a MEKdependent manner during glutamate treatment, whereas CREB expression, used as a nuclear marker, is restricted to the nucleus whatever the experimental conditions. $\boldsymbol{E}$, ERK and Elk-1 immunoblots (top row) were performed on cytoplasmic and nuclear fractions in control conditions (Cont), after $20 \mathrm{~min}$ of glutamate (Glu20'), and after 20 min of glutamate in the presence of U0126 (Glu20' $+U)$. CREB and pan-GAD immunoblots (bottom row) were used as nuclear and cytoplasmic markers, respectively. 
mate, prevented nuclear translocation of ERK and Elk-1 induced by glutamate (Fig. $2 D, E$ ).

Phosphorylation of Ser383/Ser389 residues plays a critical role in the cytoplasm to nucleus shuttling of Elk-1

Two critical residues, the Ser383 and Ser389, are phosphorylated in the C-terminal region of Elk-1 (Gille et al., 1995; Zinck et al., 1995) by activated ERKs. Although this posttranslational event is critically involved in SRE-driven gene expression, no study yet addressed its possible role in the cytoplasm to nucleus shuttling of Elk-1 in neurons. To address this, we used transfection assays with cDNA constructs encoding for HA-tagged Elk-1 either in its wild-type (Wt-Elk-1) or mutated (DN-Elk-1 and CA-Elk-1) versions. Thus, Ser383 and Ser389 were mutated into inactive (Ala383/389: dominant-negative Elk-1/DN-Elk-1) or constitutively active (Asp383/389: constitutively active Elk-1/CA-Elk-1) residues for phosphorylation (Vanhoutte et al., 2001). The subcellular localization of HA-tagged versions of Elk-1 was analyzed after HA-staining, together with MAP2 (Microtubule-Associated Protein 2, a neuritic marker) and a Hoechst labeling (Fig. $3 A$ ). In control conditions, HA-Wt-Elk-1 was found in both cytoplasmic (including neurites) and nuclear compartments (Fig. $3 A, C)$. Glutamate induced its enrichment in the nucleus, an effect abrogated by U0126 pretreatment (Fig. $3 A, C$ ). Contrasting patterns were observed with HA-DN-Elk-1 and HACA-Elk-1. Whereas HA-DN-Elk-1 showed neuritic and somatic expression whatever the treatment, HA-CA-Elk-1 was nuclear in control conditions as well as during glutamate treatment (Fig. $3 B, C$ ). U0126 pretreatment failed to affect subcellular localization of these mutated constructs (Fig. $3 B, C)$. These data demonstrate that phosphorylation of Elk-1 on Ser383/389 by activated ERKs is necessary and sufficient to drive its nuclear translocation in response to glutamate.

\section{Blocking interaction between ERKs and the DEF docking domain of Elk-1 reproduces the DN-Elk-1-induced phenotype}

Based on overexpression of Elk-1 mutants, we showed that phosphorylation of Elk-1 by activated ERKs was a necessary and sufficient condition to induce its nuclear translocation. To inhibit the phosphorylation of endogenous Elk-1, we designed a synthetic cell-penetrating peptide, named TAT-DEF-Elk-1, which mimics the interaction between activated ERKs and the DEF docking domain of Elk-1 (Fig. 4A). This DEF docking domain plays a critical role for the control of both Elk-1 binding to activated ERKs and Elk-1 phosphorylation (Fantz et al., 2001). We first verified the penetrability of this peptide into striatal neurons, using a FITC-labeled TAT-DEF-Elk-1 peptide. Striatal neurons were incubated for $1 \mathrm{~h}$ with $5 \mu \mathrm{M}$ TAT-DEF-Elk-1-FITC, followed by washing with fresh culture medium for $30 \mathrm{~min}$. In these conditions, the TAT-DEF-Elk-1-FITC entered into the majority of neurons and failed to affect the cellular morphology or DNA integrity, as shown by the MAP-2 labeling and the Hoechst counterstaining, respectively (Fig. $4 B$ ). The selective inhibition of glutamate-induced Elk-1 phosphorylation was then analyzed by Western blot using 5 or $10 \mu \mathrm{M}$ TAT-DEF-Elk-1. Whatever the doses used, TAT-DEF-Elk-1 inhibited glutamate-induced Elk-1 phosphorylation but not ERK activation (Fig. $4 C, D$ ). We used the lowest dose of TAT-DEF-Elk-1 ( $5 \mu \mathrm{M})$ for subsequent analyses. The TAT-DEF-Elk-1 peptide failed to affect the activation of MSK-1, a protein kinase activated by ERK in response to glutamate and critically involved in CREB phosphorylation (BramiCherrier et al., 2005) (Fig. 4E). Thus, as opposed to classical MEK
A

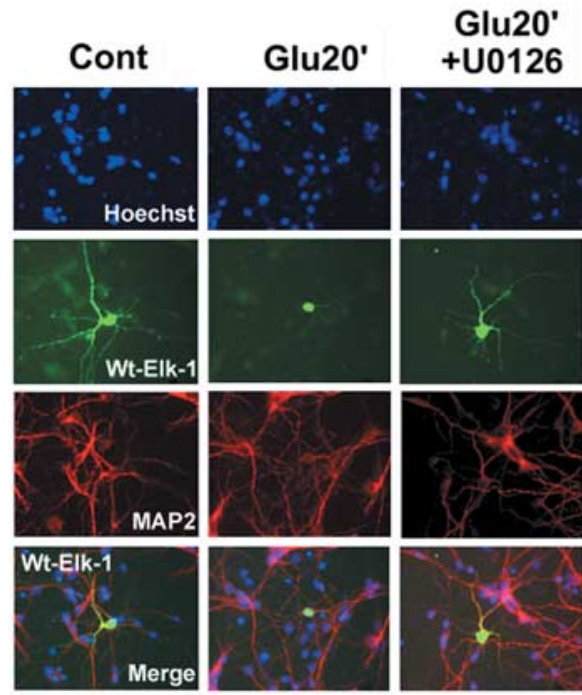

B
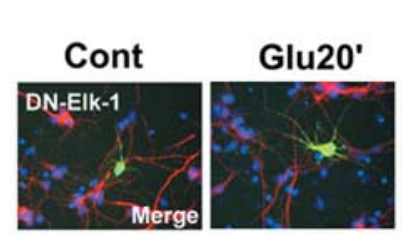

Glu20 +U0126
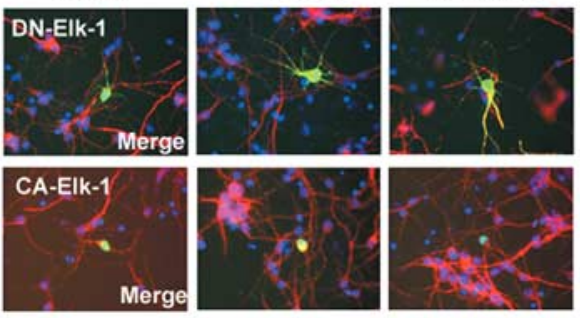

C

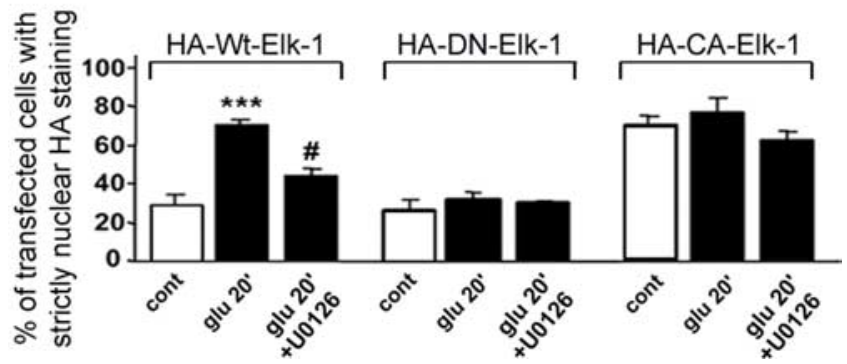

Figure 3. Phosphorylation of Elk-1 on $\mathrm{Ser}^{383}-\mathrm{Ser}^{389}$ by ERKs drives its nuclear localization. Striatal neurons were transfected at DIV6 with CDNAs encoding HA-tagged versions of wild-type (HAWt-Elk-1) (A), dominant-negative (HA-DN-Elk-1) (B, top), or constitutively active (HA-CA-Elk-1) $(\boldsymbol{B}$, bottom) forms of Elk-1. Twenty-four hours later, neurons were incubated in the absence (Cont) or presence of glutamate for $20 \mathrm{~min}\left(G l u 20^{\prime}\right)$, with or without $U 0126(10 \mu \mathrm{M})$ applied 30 min before and during glutamate application (Glu20'+U0126). A, Nuclei were stained with a Hoechst staining (blue), transfected cells were detected with anti-HA antibody (green), and the MAP2 staining (red) allows the visualization of soma and neurites. Merge (bottom) is the combination of the three channels. Note that, whereas HA-Wt-Elk-1 is expressed in both cytoplasm and nuclear compartments in control conditions, glutamate induces its nuclear translocation, an effect abrogated by $\mathrm{U} 0126$ pretreatment. $\boldsymbol{B}$, In contrast, $\mathrm{HA}-\mathrm{DN}-\mathrm{Elk}-1$ does not translocate to the nucleus during glutamate treatment (top), and CA-Elk-1 remains nuclear whatever the treatment (bottom). C, Quantifications of the percentage of neurons with a strictly nuclear staining for HA-Wt-Elk-1 (left bars), HA-DN-Elk-1 (middle bars), and HA-CA-Elk-1 (right bars) transfected cells performed from two to four independent experiments [corresponding to an average of $n=143 \mathrm{HA}-$ Wt-Elk-1, $n=112 \mathrm{HA}-\mathrm{DN}-$ Elk-1, and $n=156 \mathrm{HA}-$ CA-Elk-1 transfected neurons for each experimental conditions; statistical comparisons were performed with an unpaired Student's $t$ test ${ }^{* * *} p<0.001$ when compared with control conditions; ${ }^{\#} p<0.05$ when comparing glu $20^{\prime}$ to glu $\left.20^{\prime}+\cup 0126\right)$ ].

inhibitor treatment (Fig. 2), CREB phosphorylation was unaffected by TAT-DEF-Elk-1 (Fig. $4 F$ ).

We then used TAT-DEF-Elk-1 to analyze the subcellular localization of Elk-1 during glutamate application. Confocal mi- 
A

$\mathrm{NH} 2$

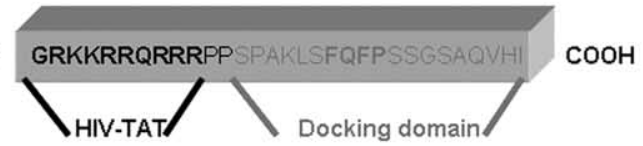

B
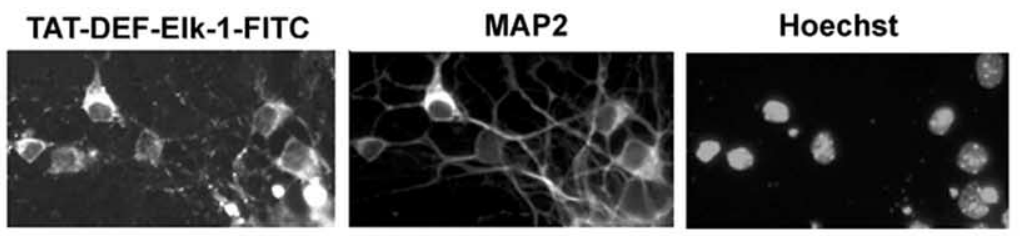

C
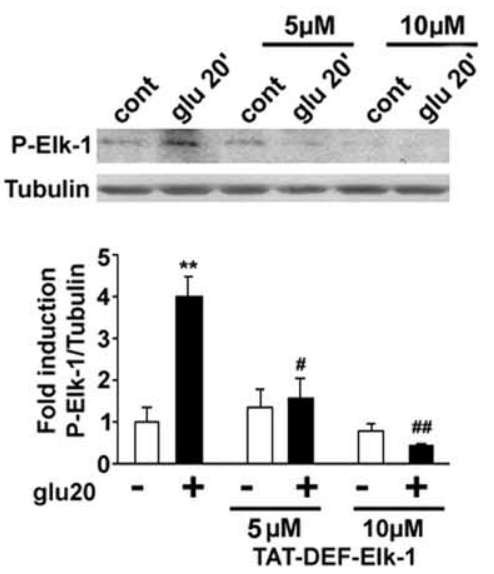

$\mathbf{E}$
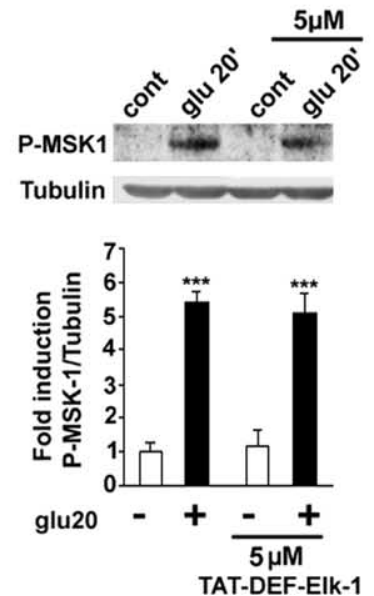

D
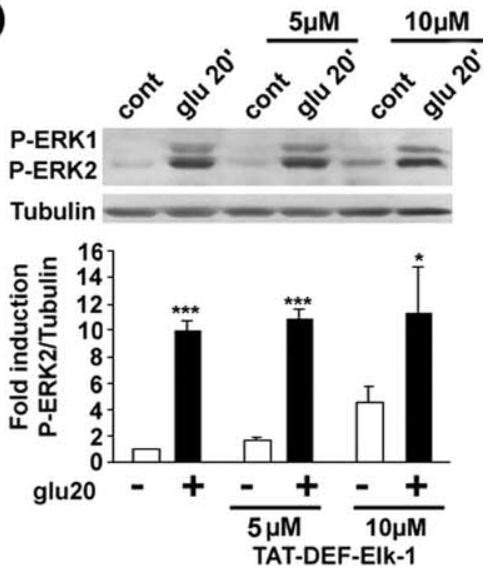

$\mathbf{F}$
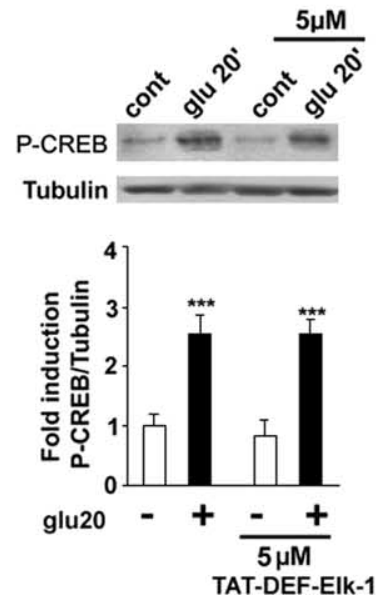

Figure 4. TAT-DEF-Elk-1 specifically inhibits Elk-1 phosphorylation. $A$, Diagram depicting the structure of TAT-DEF-Elk-1 peptide; the N-terminal part is composed of a fragment of HIV-TAT linked to the Elk-1 DEF docking site for ERK. The DEF domain includes the minimum consensus (FQFP) as well as flanking sequence of Elk-1. These two domains are separated by two proline residues to allow flexibility of the peptide. This cell permeable peptide (via HIV-TAT) has been designed to specifically alter Elk-1 binding to activated ERK1/2 via its DEF domain. $\boldsymbol{B}$, Neurons were incubated with a FITC-labeled TAT-DEF-Elk-1 (5 $\mu \mathrm{m})$ for $1 \mathrm{~h}$ as indicated in Materials and Methods. Intracellular accumulation of TAT-DEF-Elk-1-FITC peptide (in green) has been analyzed by confocal microscopy. MAP2 (red) and Hoechst (blue) costaining show no alteration of cellular morphology and a lack of toxicity of the TAT-DEF-Elk-1-FITC peptide, respectively. Neurons were incubated or not with the TAT-DEF-Elk-1 peptide at 5 or $10 \mu \mathrm{m}$ before an incubation with (glu 20') or without (cont) glutamate for 20 min. Activation of Elk-1 (C) and ERK (D) was analyzed by Western blot using phospho-specific antibodies. Quantifications of Elk-1 ( $\boldsymbol{C}$, bottom) and ERK ( $\boldsymbol{D}$, bottom) phosphorylation were performed from three independent experiments. Downstream from ERK, the effect of TAT-DEF-Elk-1 (5 $\mu \mathrm{m})$ on glutamateinduced activation of MSK-1 $(\boldsymbol{E})$ and CREB $(\boldsymbol{F})$ was studied by Western blot and quantified from three independent experiments. Note that TAT-DEF-Elk-1 specifically inhibits glutamate-induced Elk-1 activation without interfering with ERK, MSK-1, or CREB phosphorylation. Statistics were performed with unpaired Student's $t$ test $\left({ }^{*} p<0.05,{ }^{* *} p<0.01,{ }^{* * *} p<0.001\right.$ when compared with control conditions; ${ }^{\#} p<0.05,{ }^{\# \#} p<0.01$ when comparing glutamate treatment in the presence or not of TAT-DEF-Elk-1).

croscopy analyses (Fig. 5A) showed no nuclear translocation of Elk-1 during glutamate treatment in the presence of TAT-DEF-Elk-1. These data were confirmed biochemically after cellular fractionations (Fig. 5B). This lack of nuclear translocation correlated nicely with the absence of glutamate-mediated Elk-1 phosphorylation in the nucleus (Fig. 5C). In contrast, glutamate-induced ERK nuclear translocation (Fig. 5B) and activation (Fig. $5 D$ ) were not affected by the TATDEF-Elk-1. As expected, the phosphorylation levels of some important downstream nuclear targets of ERK (Brami-Cherrier et al., 2005) were not affected by the peptide, including MSK-1, CREB, and histone H3 (Fig. $5 E-G$ ).

Comparative effects of MEK inhibitor and TAT-DEF-Elk-1 peptide on glutamate-induced gene expression TAT-DEF-Elk-1 was designed to inhibit Elk-1 phosphorylation induced by activated ERKs, without interfering with MSK1/CREB module or chromatin remodeling via histone $\mathrm{H} 3$ phosphorylation. We thus wished to compare the effects of TAT-DEF-Elk-1 and a global MEK inhibitor (U0126) on glutamate-induced gene expression. For this purpose, we chose IEGs that are controlled by the ERK cascade via their SRE and/or CRE binding domains (namely c-Fos, Zif268 and JunB) or via histone $\mathrm{H} 3$ phosphorylation (namely c-Jun) (supplemental Fig. 1, available at www.jneurosci.org as supplemental material) (Brami-Cherrier et al., 2007). The U0126 compound had a profound inhibitory role on glutamate-induced c-Fos, Zif268 and JunB induced expression (Fig. $6 A-D$ ) and a partial, but significant, inhibitory action on c-Jun expression (Fig. 6E). The effects of TAT-DEF-Elk-1 treatment on gene expression were statistically different from those observed with U0126 (Fig. $6 A-E$ ), with a significant inhibition of c-Fos, Zif268 and JunB but no effect on c-Jun expression. These observations were specifically attributable to the inhibition of Elk-1 phosphorylation because we failed to detect any effect of a scramble TATDEF-Elk-1 peptide. Transfection assays using the DN-Elk-1 mutant also showed partial blockade of glutamate-induced c-Fos, Zif268 and JunB, but not c-Jun, expression (supplemental Fig. 2, available at www.jneurosci.org as supplemental material). Although overexpression of CAElk-1 was not sufficient to increase the expression of basal IEGs, it produced increases of glutamate-induced expression of Zif268, an IEG baring multiple SRE sites 
A

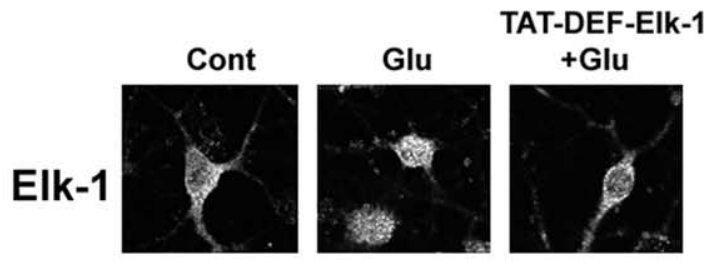

B

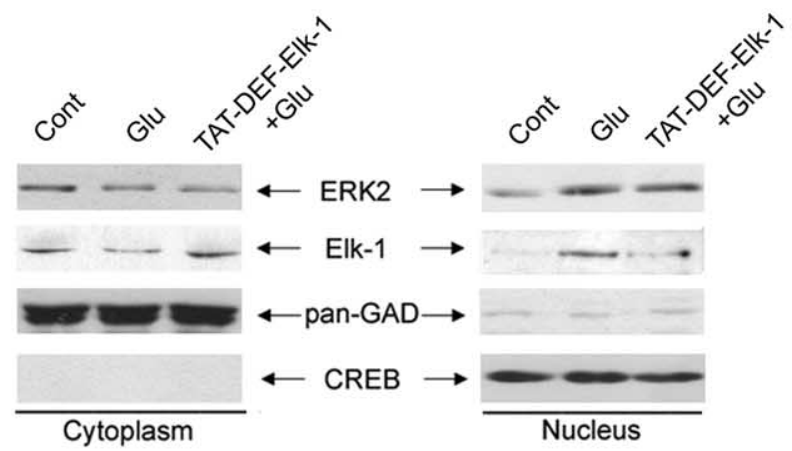

C

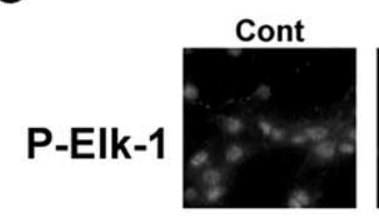

D

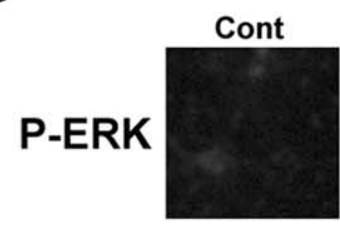

E

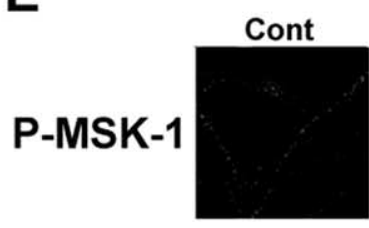

F

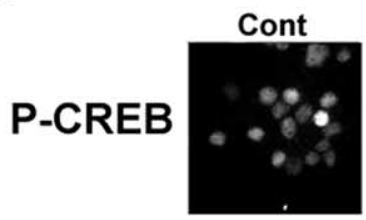

G

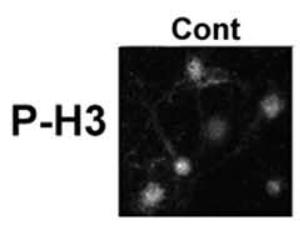

TAT-DEF-Elk-1
TAT-DEF-Elk-1
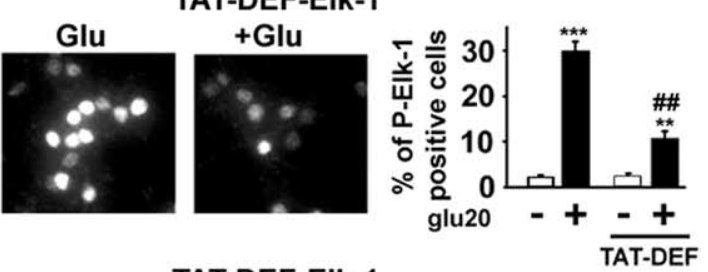

TAT-DEF-Elk-1
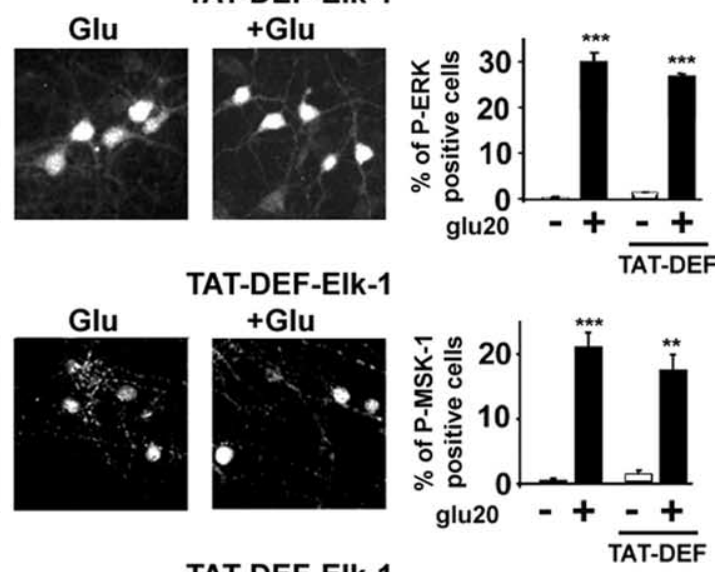

TAT-DEF-Elk-1
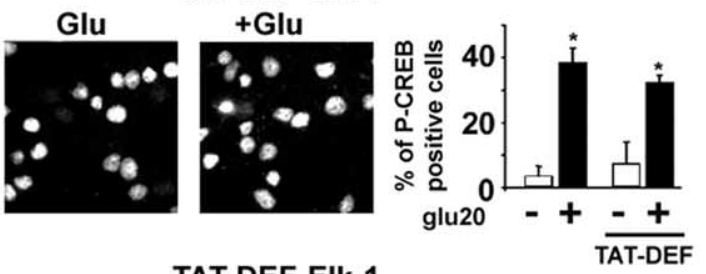

TAT-DEF-Elk-1
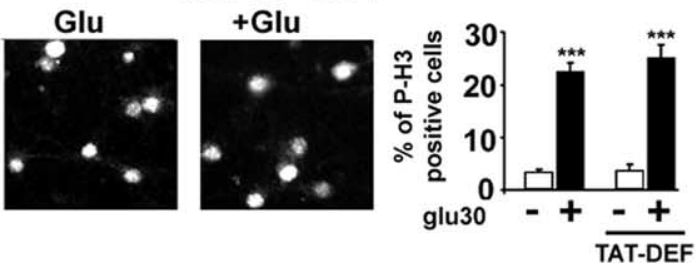

Figure 5. TAT-DEF-Elk-1 prevents both nuclear translocation and phosphorylation of Elk-1 without affecting glutamateinduced ERK phosphorylation other targets. $A$, Effect of TAT-DEF-Elk-1 on the cytoplasm-to-nucleus trafficking of Elk-1. Representative confocal sections of endogenous Elk-1 proteins. $\boldsymbol{B}$, ERK and Elk-1 immunoblots (top) were performed on cytoplasmic and nuclear fractions in control conditions (Cont), after 20 min of glutamate (Glu), and after 20 min of glutamate in the presence of the

in its promoter (supplemental Fig. 2, available at www.jneurosci.org as supplemental material). Thus, although not sufficient, ERK-mediated phosphorylation of Elk-1 seems to be a prerequisite for proper glutamate-induced gene regulations in neurons.

Our data provide evidence that Elk-1 phosphorylation on Ser 383/389 plays a dual role in neurons, and triggers both its nuclear translocation and SRE-driven gene expression.

Role of ERK-dependent Elk-1 activation in neuronal differentiation

Depending on the spatiotemporal profile of ERK activation, the phosphorylation of Elk-1 has been reported to be associated with multiple phenotypes, including differentiation and cell proliferation (Hipskind et al., 1994a,b; York et al., 1998). In PC12 cells, we previously demonstrated that, NGF-induced neuronal differentiation was linked to the activation of a nuclear, truncated, isoform of Elk-1 called sElk-1 (Vanhoutte et al., 2001). However, no study yet addressed the role of Elk-1 phosphorylation and nuclear translocation, in neuronal differentiation of primary cultured neurons. To address this aspect, we compared the effects of TATDEF-Elk-1 and U0126, in dendritic and axonal elongation during spontaneous differentiation of striatal neurons in vitro. Because this set of experiments required long lasting incubations of the peptide (from DIV 3 to DIV 7), we used a more stable version composed of the amino acids in their D-conformation. This D-peptide had the same biochemical properties as the peptide synthesized with amino acids in their natural conformations (data not shown). After pharmacological treatments, cells were transfected with GFP to visualize the morphology of

\footnotetext{
$\leftarrow$

TAT-DEF-Elk-1 peptide (TAT-DEF-Elk-1+Glu). CREB and pan-GAD immunoblots (bottom) were used as nuclear and cytoplasmic markers, respectively. Note that glutamateinduced Elk-1, but not ERK2, nuclear translocation is inhibited by the TAT-DEF-Elk-1 peptide. $\mathbf{C}-\mathbf{G}$, Immunocytochemistry was performed for P-Elk-1, P-ERK, P-MSK-1, P-CREB, and $\mathrm{P}$-H3 in the absence or presence of TAT-DEF-Elk-1 peptide.C, Note the strong decrease of glutamate-induced P-Elk-1positive cells in presence of TAT-DEF-Elk-1 and the lack of effect of this peptide on glutamate-induced P-ERK (D), P-MSK-1 (E), P-CREB $(\boldsymbol{F})$, and P-H3 (G) signals. Statistical analyses were performed from three independent experiments using unpaired Student's $t$ test $\left({ }^{*} p<0.05,{ }^{* *} p<\right.$ $0.01,{ }^{* * *} p<0.001$ when comparing controls with glutamate treatment; ${ }^{\# \#} p<0.01$ when comparing glutamate treatment in the presence or not of TAT-DEF-Elk-1).
} 
A

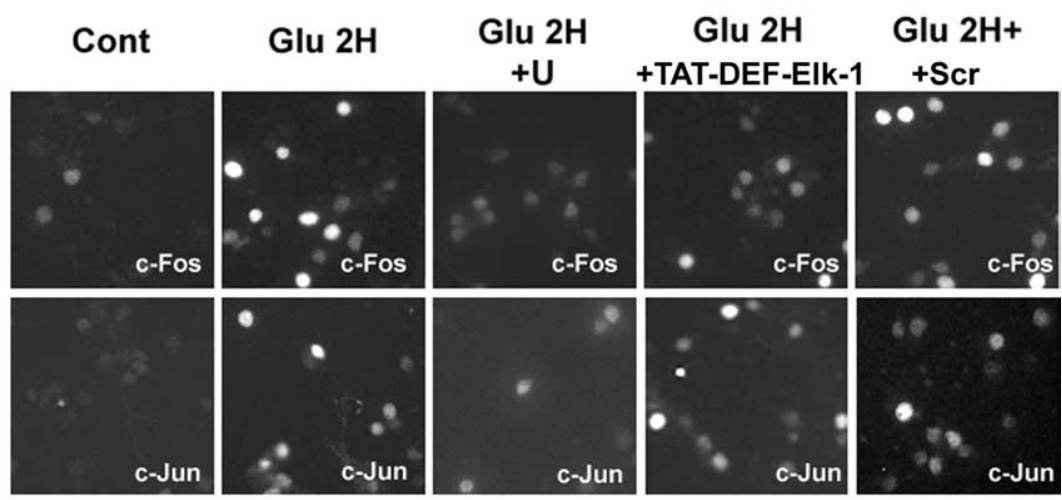

B

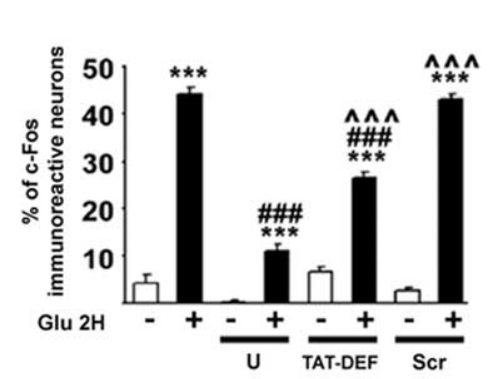

D

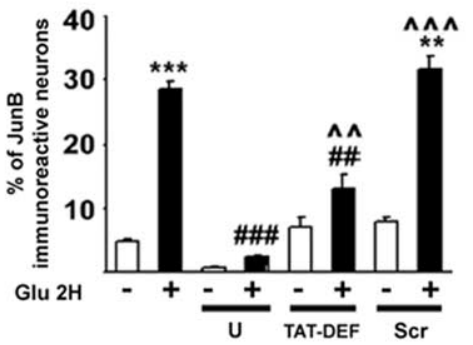

C

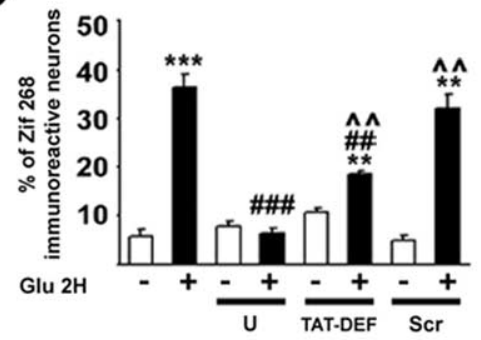

E

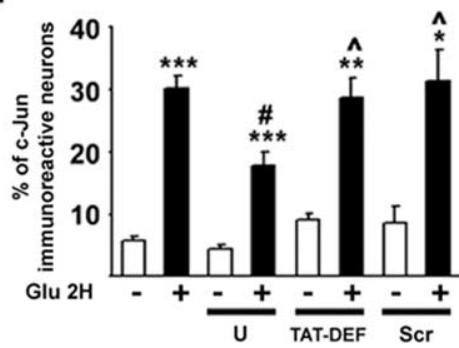

Figure 6. Comparative effects of the MEK inhibitor (U0126) and the TAT-DEF-Elk-1 peptide on SRE-driven gene regulation. Immunocytochemical detection of c-Fos $(\boldsymbol{A})$, Zif268, JunB, and c-Jun were performed from primary striatal neurons in control conditions and after $2 \mathrm{~h}$ of incubation with glutamate application in the absence (Glu 2H) or presence of U0126 (U), TAT-DEFElk-1 (5 $\mu \mathrm{m}$; TAT-DEF), and scramble version of TAT-DEF-Elk-1 peptide ( $5 \mu \mathrm{m} ; \mathrm{Scr})$. Quantification of the percentage of c-Fos $(\boldsymbol{B})$, Zif268 (C), JunB (D), and C-Jun (E) positive striatal cells were performed from three independent experiments. Statistical comparisons were performed with unpaired Student's $t$ test $\left({ }^{*} p<0.05,{ }^{* *} p<0.01,{ }^{* *} p<0.001\right.$ when comparing glutamate with

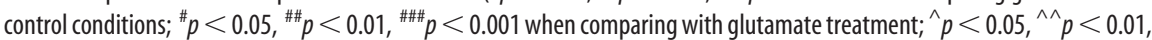

$\wedge_{\wedge} p<0.001$ when comparing with glutamate treatment in the presence of U0126). Note that TAT-DEF-Elk-1 partially inhibits c-Fos, Zif268, and JunB but not c-Jun expression.

neurons, and dendrites were labeled by immunocytochemistry with a MAP2 antibody (Fig. 7A). The length of the axon, which was considered as the longest and thinnest extension, as well as the mean dendritic length per cell, was analyzed. Inhibition of ERK-dependent Elk-1 phosphorylation with TAT-DEF-Elk-1 reduced the dendritic outgrowth to the same extent as the classical MEK inhibitor, U0126 (Fig. 7B, left). With regard to the axon outgrowth, both TAT-DEF-Elk-1 and U0126 tend to reduce the mean axonal length. However, statistical analysis revealed a difference between these two compounds because the global MEK inhibitor significantly reduced the axon length whereas the TAT-DEFElk-1 did not (Fig $7 B$, right). Whereas the global inhibition of the ERK pathway with U0126 revealed a toxic role, the selective inhibition of Elk-1 phosphorylation with the TAT-DEF-Elk-1 did not trigger neuronal death (Fig. 7E).
To further substantiate that the effects of the TAT-DEF-Elk-1 peptide on dendritic elongation was attributable to the suppression of Elk-1 phosphorylation, we used the cDNA constructs encoding for the mutated versions of Elk-1 (DN-Elk-1 and CA-Elk-1). These constructs were tagged with Cherry, and cotransfected with GFP on DIV1 in striatal neurons (Fig. $7 C)$. As controls, striatal neurons were transfected with GFP alone, treated or not with the TAT-DEF-Elk-1 peptide (Fig. $7 D)$. Dendritic elongation measured on DIV7 showed a significant reduction in DN-Elk-1 transfected neurons when compared with GFP alone (Fig. 7C,D). In contrast, CA-Elk-1-containing neurons showed increased dendritic length (Fig. $7 C, D)$. Neither DN- nor CA-Elk-1 constructs did alter neuronal cell survival when compared with GFP alone transfected neurons (Fig. 7E) Importantly, DN-Elk-1 containing neurons and neurons treated with TAT-DEF-Elk-1 showed no significant difference in the mean dendritic length, thus indicating that the effects of the peptide was merely attributable to the suppression of Elk-1 phosphorylation (Fig. 7C,D).

A recent study of forebrain-specific-srf null mice's phenotype pointed out the critical role of the Elk-1 protein partner SRF in neuronal differentiation. Hippocampal neurons of srf-deficient mice showed a decreased expression of Actin mRNA associated with severe impairments of neuritic outgrowth and cytoskeleton dynamics (Knöll et al., 2006). Because Elk-1 activation positively regulates SRF expression levels (Kasza et al., 2005), we analyzed the effect of chronic inhibition of Elk-1 phosphorylation with the TAT-DEF-Elk-1 or U0126 on SRF and Actin expression levels. Immunoblot experiments, showed a significant decrease in SRF and Actin protein expression levels with both treatments (Fig. 8A). To assess the impact of TATDEF-Elk-1 and U0126 on the cytoskeleton dynamic, costaining of F-actin, with rhodamine-coupled phalloidin, and tubulin was performed. We observed that neurons bore growth cones with well structured filopodia in control conditions (Fig. $8 \mathrm{~B}$, left). In contrast, incubation with the TAT-DEF-Elk-1 peptide led to a collapse of growth cones, as evidenced by the disorganized actin cytoskeleton (Fig. $8 \mathrm{~B}$, middle). In the presence of the global ERK inhibitor a complete breakdown of F-actin was found, indicating a drastic impairment of the cytoskeleton dynamics (Fig. $8 B$, left). Altogether, these data show a specific role of Elk-1 phosphorylation in the control of the cytoskeleton dynamics in neuronal cells.

\section{Discussion}

In the present study, we provide the first evidence that the transcription factor Elk-1 translocates from the cytoplasm to the nu- 
cleus during phosphorylation in neurons. Phosphorylation of Ser383/389 by activated ERKs plays a dual role in Elk- 1 functions, and controls both nuclear translocation and SRE-driven gene expression. Using dominant-negative Elk-1 mutants or a peptide inhibiting the DEF docking domain of Elk-1 to ERKs, we unraveled a fundamental role of Elk-1 phosphorylation in dendritic elongation, an effect associated with decreased expression levels of Actin and SRF as well as severe impairments of the cytoskeleton dynamics.

From overexpression assays in HeLa cells, Elk-1 was initially described as a nuclear transcription factor (Pingoud et al., 1994). We previously demonstrated that this scenario was different in cerebral tissues in which the endogenous Elk-1 was expressed and activated in both the cytoplasm and the nucleus (Sgambato et al., 1998a,b). Herein, we reinforce these observations and provide evidences for an enrichment of Elk-1 expression in the nucleus by activated ERKs, in vivo, in response to cocaine, and in vitro during glutamate stimulation. Importantly, the nuclear translocation of Elk-1 is associated with SRE-driven gene regulation. Thus, similarly to other transcription factors, including STAT 1 (Meyer et al., 2003), NF-AT4 (Shibasaki et al., 1996), and NF-Kappa-B (Mikenberg et al., 2006), Elk-1 undergoes a nuclear translocation required for its role in transcription. The nuclear transport of proteins that are larger than $60 \mathrm{kDa}$, such as Elk-1, are facilitated by their interaction with importins $(\alpha / \beta)$ proteins. In this case, the entry to the nucleus is an active process and requires a NLS sequence, which is recognized by the nuclear import machinery (Weis et al., 2003). Importantly, during activation by ERKs, Elk-1 undergoes important conformational changes that increase its interaction with DNA at the SRE site (Yang et al., 1999). One can speculate that such conformational changes could unmask NLS present in Elk-1 and enable its interaction with importins (or karyopherins), as previously described for the transcription factor STAT1 (Fagerlund et al., 2002; McBride et al., 2002).

The phosphorylation-dependent trafficking of Elk-1 to the nucleus is a major observation because, within the cytoplasm, Elk-1 is associated with the mitochondrial PTP (Permeability Transition Pore complex), an interaction that increases in the context of proapoptotic treatments (Barrett and al., 2006b). Furthermore, restricting Elk-1 transcription and translation in neurites, using a focal transfection procedure named "phototransfection", produces cell death, whereas its introduction and translation in cell bodies does not (Barrett et al., 2006a). Altogether, these data suggest that Elk-1 plays a dual role in neuronal cells, which is clearly driven by its subcellular localization and posttranslational modifications.

Other posttranslational modifications have been described for Elk-1. In this way, its SUMOylation on 3 critical residues (Lys230/ 249/254), results in a more rapid nuclear translocation of Elk-1 (Salinas et al., 2004). Furthermore, a dynamic interplay between the activating ERK/MAP kinase pathway and the repressive SUMO pathway has been described, whereby ERK activation leads to both phosphorylation of Elk-1 and loss of SUMO conjugation (Yang et al., 2003). Such interplay could be critically involved in the shuttling of Elk-1, with a role of phosphorylation in the nuclear import of Elk-1, whereas SUMOylation could favor its export from the nucleus.

Although Elk-1 possesses two specific docking domains for activated ERK, the D (or DEJL) and the DEF (or FxFP) domains (Jacobs et al., 1999; Sharrocks et al., 2000), recent evidence involves the DEF domain as the most important motif for its phosphorylation on Serine 383 (Fantz et al., 2001). Furthermore, mu-

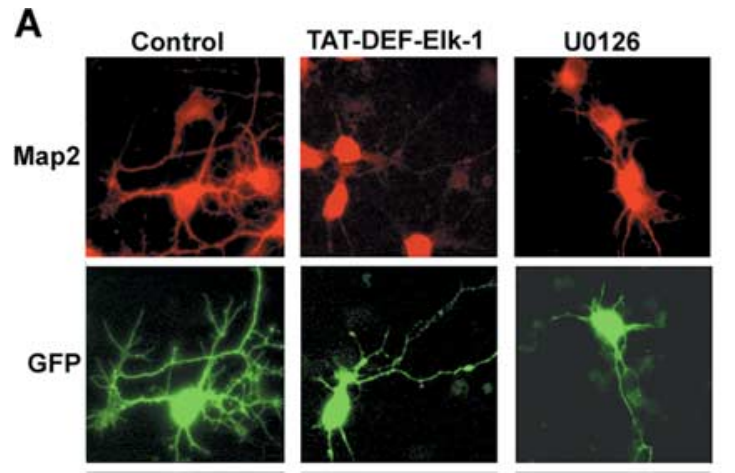

B.
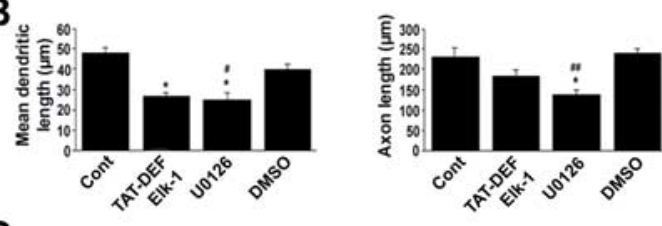

C

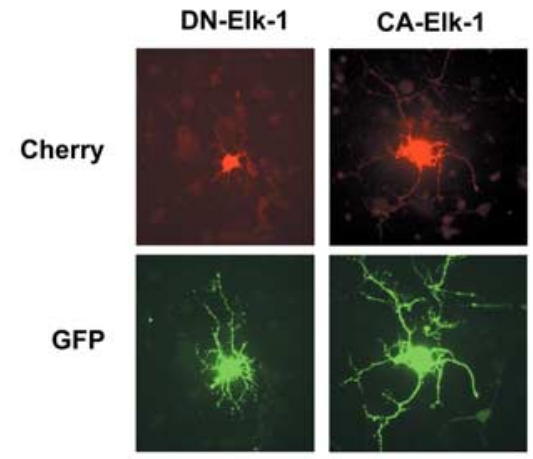

D

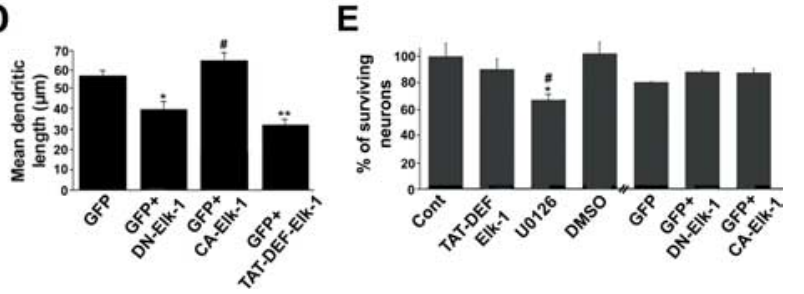

Figure 7. Role of Elk-1 phosphorylation in neuritic elongation. $\boldsymbol{A}$, Striatal neurons were incubated in the absence (control; left column) or presence of $5 \mu \mathrm{m}$ TAT-DEF-Elk-1 peptide in its D-enantiomer form (middle column), U0126 (10 $\mu \mathrm{m}$; right panels) at DIV 3 and left in the medium containing the various drugs until DIV7. At DIV6, neurons were transfected with a GFP construct (green; bottom row). At DIV7, cells were fixed and an MAP2 staining (red; top panels) was performed by indirect immunofluorescence. Both labeling allows the visualization of the cellular morphology. B, Histograms show mean dendritic (left) and axonal (right) length obtained from four independent experiments. Statistical comparisons were performed with unpaired Student's $t$ test ${ }^{*} p<0.05$ when comparing with the control group; ${ }^{*} p<0.05$ when comparing with the DMSO group; $\#$ $p<0.01)$. C, Cherry-tagged DN-Elk-1 or CA-Elk-1 constructs (top row, red) were cotransfected with GFP (bottom row, green) in striatal neurons on DIV1, and the morphology was analyzed on DIV7 on the basis of GFP staining. D, Histograms show mean dendritic length of striatal neurons transfected with GFP alone, cotransfected with Cherry-DN- or CA-Elk-1, or treated with TAT-DEF-Elk-1. Quantification and statistics were performed as in $\boldsymbol{B}\left({ }^{*} p<0.05,{ }^{* *} p<0.01\right.$ when comparing with the control group; ${ }^{*} p<0.05$ when comparing the (A-Elk-1 to the DN-Elk-1 group). E, Histograms showing neuronal survival in response to TAT-DEF-Elk-1, U0126, or DMSO treatments when compared with controls (left) and in striatal neurons transfected with GFP alone, cotransfected with Cherry-DN- or CA-Elk-1 (right). Quantification and statistics were performed as in $B)\left({ }^{*} p<0.05\right.$ when comparing with the control group; ${ }^{*} p<0.05$ when comparing the U0126 with the TAT-DEF-Elk-1 group).

tating Ser383 into an inactive residue blocks Elk-1 transcriptional activity (Gille et al., 1995). To specifically alter ERK-mediated Elk-1 phosphorylation, we designed a cell-penetrating peptide, the TAT-DEF-Elk-1 peptide, that mimics the DEF domain of 
A

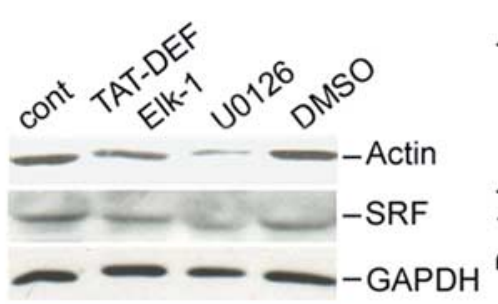

B
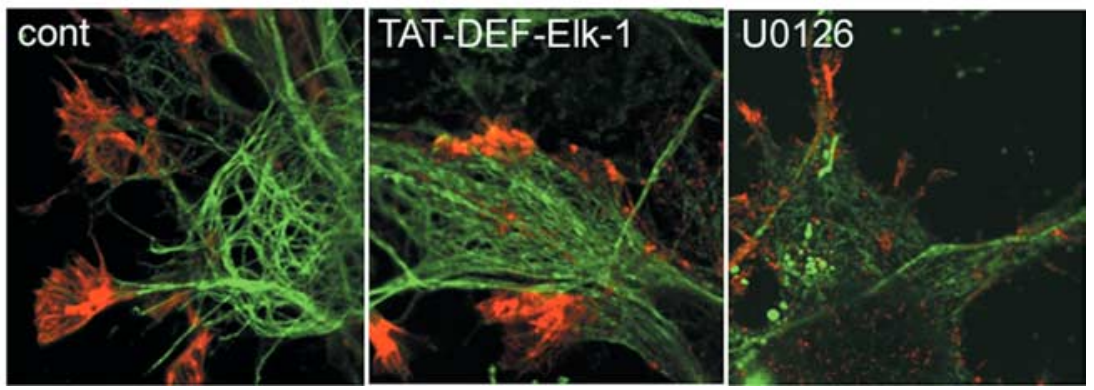

Figure 8. Elk-1 activation controls SRF and actin expression as well as the cytoskeleton dynamics. $A$, Immunoblots performed with antibodies directed against actin (top) and SRF (middle) proteins at DIV7 on cultured neurons treated from DIV3 to DIV7 with the TAT-DEF-Elk-1, U0126, or DMSO. The GAPDH immunoblots (bottom) serves as a loading control. Quantifications of SRF (white bars) and actin (black bars) expression levels were performed from three independent experiments (left panel; ${ }^{*} p<0.05$, unpaired Student's $t$ test when comparing with the control group). $\boldsymbol{B}$, Costaining of F-actin (revealed with rhodamine-coupled phalloidin) and tubulin (detected by indirect fluorescence with an FITC-labeled secondary antibody) performed at DIV7 from neurons in control conditions and after $4 \mathrm{~d}$ of incubations with the indicated drugs.

Elk-1. As expected, this peptide blocked glutamate-induced Elk-1 phosphorylation and nuclear translocation, without interfering with ERK activation and nuclear translocation. We observed that some important nuclear events driven by activated ERKs were not affected by the peptide, namely phosphorylation of MSK-1, CREB and histone H3. MSK-1 is a direct nuclear target of ERK with no DEF domain (Sharrocks et al., 2000), critically involved in both CREB and histone $\mathrm{H} 3$ phosphorylation in striatal neurons (Brami-Cherrier et al., 2005, 2007). As a consequence, the pattern of gene expression induced by glutamate was differently affected by the TAT-DEF-Elk-1 peptide compared with a classical MEK inhibitor, which had a more widespread effect. Thus, the only IEGs that were downregulated by the TAT-DEF-Elk-1 peptide were those carrying SRE-sites in their promoter (c-Fos, JunB, and Zif268), whereas the global MEK inhibitor also blocked SREindependent events, such as c-Jun induction via histone $\mathrm{H} 3$ phosphorylation (Brami-Cherrier et al., 2007).

The role of Elk-1 in neuronal differentiation has been proposed from NGF-treated PC12 cells. We originally described that overexpression of a nonphosphorylatable version of Elk-1 (DNElk-1) in PC12 cells blocked their differentiation induced by NGF (Vanhoutte et al., 2001). More recently, PC12 overexpressing a non-SUMOylable mutant of Elk-1, showed a facilitation of neuronal differentiation (Salinas et al., 2004). In the present work, we extend these observations, and show that chronic inhibition of Elk-1 phosphorylation with the TAT-DEF-Elk-1 peptide inhibits spontaneous dendritic outgrowth. Dendritic elongation was inhibited to the same extent with the DN-Elk-1 cDNA construct, indicating that the effects of the peptide was merely attributable to the suppression of Elk-1 phosphorylation. Importantly, inhibition of dendritic elongation induced by TAT-DEF-Elk-1 was associated with an impairment of the cytoskeleton dynamics and a downregulation of SRF and Actin expression. SRF activity is intimately linked to the cytoskeleton dynamics, in particular to the polymerization of G-actin into F-actin (Sotiropoulos et al., 1999). The downregulation of SRF expression found during TAT-DEF-Elk-1 treatment, is in agreement with previous reports, showing that Elk-1 activation positively regulates SRF expression (Kasza et al., 2005). The phenotype observed in the present study correlates with that recently described from a conditional forebrain-specific srf knockout mice. In this work (Knöll et al., 2006) the authors observed shorter neuritic length and alteration of the cytoskeleton dynamics in hippocampal neurons from deficient mice. These events were associated, similarly to the chronic treatment with TAT-DEF-Elk-1, with impairments of growth cones dynamics and downregulation of Actin mRNA levels. We propose that phosphorylation of Elk-1 is an upstream event in a complex genetic program that controls neuronal differentiation and that TAT-DEF-Elk-1 is a powerful tool to analyze the role of Elk-1 in this process during development or neuronal plasticity, for example in response to cocaine.

\section{References}

Atkins CM, Selcher JC, Petraitis JJ, Tracks JM, Sweatt JD (1998) The MAPK cascade is required for mammalian associative learning. Nat Neurosci 1:602-609.

Barrett LE, Sul JY, Takano H, Van Bockstaele EJ, Haydon PG, Eberwine JH (2006a) Region-directed phototransfection reveals the functional significance of a dendritically synthesized transcription factor. Nat Methods 3:455-460.

Barrett LE, Van Bockstaele EJ, Sul JY, Takano H, Haydon PG, Eberwine JH (2006b) Elk-1 associates with the mitochondrial permeability transition pore complex in neurons. Proc Natl Acad Sci USA 103:5155-5160.

Brami-Cherrier K, Valjent E, Garcia M, Pages C, Hipskind RA, Caboche J (2002) Dopamine induces a PI3-kinase-independent activation of Akt in striatal neurons: a new route to cAMP response element-binding protein phosphorylation. J Neurosci 22:8911-8921.

Brami-Cherrier K, Valjent E, Herve D, Darragh J, Corvol JC, Pages C, Arthur SJ, Girault JA, Caboche J (2005) Parsing molecular and behavioral effects of cocaine in mitogen- and stress-activated protein kinase-1deficient mice. J Neurosci 25:11444-11454.

Brami-Cherrier K, Lavaur J, Pagès C, ARthur JS, Caboche J (2007) Glutamate induces histone $\mathrm{H} 3$ phosphorylation but not acetylation in striatal neurons: role of mitogen and stress-activated kinase-1 (MSK-1). J Neurochem 101:697-708.

Buchwalter G, Gross C, Wasylyk B (2004) Ets ternary complex transcription factors. Gene 324:1-14.

Fagerlund R, Melen K, Kinnunen L, Julkunen I (2002) Arginine/lysine-rich nuclear localization signals mediate interactions between dimeric STATs and importin alpha 5. J Biol Chem 277:30072-30078.

Fantz DA, Jacobs D, Glossip D, Kornfeld K (2001) Docking sites on substrate proteins direct extracellular signal-regulated kinase to phosphorylate specific residues. J Biol Chem 276:27256-27265.

Gille H, Kortenjann M, Thomae O, Moomaw C, Slaughter C, Cobb MH, Shaw PE (1995) ERK phosphorylation potentiates Elk-1-mediated ternary complex formation and transactivation. EMBO J 14:951-962. 
Hipskind RA, Baccarini M, Nordheim A (1994a) Transient activation of RAF-1, MEK, and ERK2 coincides kinetically with ternary complex factor phosphorylation and immediate-early gene promoter activity in vivo. Mol Cell Biol 14:6219-6231.

Hipskind RA, Buscher D, Nordheim A, Baccarini M (1994b) Ras/MAP kinase-dependent and -independent signaling pathways target distinct ternary complex factors. Genes Dev 8:1803-1816.

Jacobs D, Glossip D, Xing H, Muslin AJ, Kornfeld K (1999) Multiple docking sites on substrate proteins form a modular system that mediates recognition by ERK MAP kinase. Genes Dev 13:163-175.

Janknecht R, Zinck R, Ernst WH, Nordheim A (1994) Functional dissection of the transcription factor Elk-1. Oncogene 9:1273-1278.

Kasza A, O'Donnell A, Gascoigne K, Zeef LA, Hayes A, Sharrocks AD (2005) The ETS domain transcription factor Elk-1 regulates the expression of its partner protein, SRF. J Biol Chem 280:1149-1155.

Knöll B, Kretz O, Fiedler C, Alberti S, Schutz G, Frotscher M, Nordheim A (2006) Serum response factor controls neuronal circuit assembly in the hippocampus. Nat Neurosci 9:195-204.

McBride KM, Banninger G, McDonald C, Reich NC (2002) Regulated nuclear import of the STAT1 transcription factor by direct binding of importin-alpha. EMBO J 21:1754-1763.

Meyer T, Marg A, Lemke P, Wiesner B, Vinkemeier U (2003) DNA binding controls inactivation and nuclear accumulation of the transcription factor Stat1. Genes Dev 17:1992-2005.

Mikenberg I, Widera D, Kaus A, Kaltschmidt B, Kaltschmidt C (2006) TNFalpha mediated transport of NF-kappaB to the nucleus is independent of the cytoskeleton-based transport system in non-neuronal cells. Eur J Cell Biol 85:529-536.

Pingoud V, Zinck R, Hipskind RA, Janknecht R, Nordheim A (1994) Heterogeneity of ternary complex factors in HeLa cell nuclear extracts. J Biol Chem 269:23310-23317.

Salinas S, Briancon-Marjollet A, Bossis G, Lopez MA, Piechaczyk M, JarielEncontre I, Debant A, Hipskind RA (2004) SUMOylation regulates nucleo-cytoplasmic shuttling of Elk-1. J Cell Biol 165:767-773.

Sgambato V, Vanhoutte P, Pages C, Rogard M, Hipskind R, Besson MJ, Caboche J (1998a) In vivo expression and regulation of Elk-1, a target of the extracellular-regulated kinase signaling pathway, in the adult rat brain. J Neurosci 18:214-226.
Sgambato V, Pages C, Rogard M, Besson MJ, Caboche J (1998b) Extracellular signal-regulated kinase (ERK) controls immediate early gene induction on corticostriatal stimulation. J Neurosci 18:8814-8825.

Sharrocks AD, Yang SH, Galanis A (2000) Docking domains and substratespecificity determination for MAP kinases. Trends Biochem Sci $25: 448-453$.

Shibasaki F, Price ER, Milan D, McKeon F (1996) Role of kinases and the phosphatase calcineurin in the nuclear shuttling of transcription factor NF-AT4. Nature 382:370-373.

Sotiropoulos A, Gineitis D, Copeland J, Treisman R (1999) Signal-regulated activation of serum response factor is mediated by changes in actin dynamics. Cell 98:159-169.

Valjent E, Corvol JC, Pages C, Besson MJ, Maldonado R, Caboche J (2000) Involvement of the extracellular signal-regulated kinase cascade for cocaine-rewarding properties. J Neurosci 20:8701-8709.

Vanhoutte P, Barnier JV, Guibert B, Pages C, Besson MJ, Hipskind RA, Caboche J (1999) Glutamate induces phosphorylation of Elk-1 and CREB, along with c-fos activation, via an extracellular signal-regulated kinasedependent pathway in brain slices. Mol Cell Biol 19:136-146.

Vanhoutte P, Nissen JL, Brugg B, Gaspera BD, Besson MJ, Hipskind RA, Caboche J (2001) Opposing roles of Elk-1 and its brain-specific isoform, short Elk-1, in nerve growth factor-induced PC12 differentiation. J Biol Chem 276:5189-5196.

Weis K (2003) Regulating access to the genome: nucleocytoplasmic transport throughout the cell cycle. Cell 112:441-451.

Yang SH, Shore P, Willingham N, Lakey JH, Sharrocks AD (1999) The mechanism of phosphorylation-inducible activation of the ETS-domain transcription factor Elk-1. EMBO J 18:5666-5674

Yang SH, Jaffray E, Hay RT, Sharrocks AD (2003) Dynamic interplay of the SUMO and ERK pathways in regulating Elk-1 transcriptional activity. Mol Cell 12:63-74.

York RD, Yao H, Dillon T, Ellig CL, Eckert SP, McCleskey EW, Stork PJ (1998) Rap1 mediates sustained MAP kinase activation induced by nerve growth factor. Nature 392:622-626.

Zinck R, Cahill MA, Kracht M, Sachsenmaier C, Hipskind RA, Nordheim A (1995) Protein synthesis inhibitors reveal differential regulation of mitogen-activated protein kinase and stress-activated protein kinase pathways that converge on Elk-1. Mol Cell Biol 15:4930-4938. 